\title{
TEMPERATURE EFFECT ON FREQUENCIES OF A TAPERED TRIANGULAR PLATE
}

\author{
Akash Khodiya, Amit Sharma \\ Department of Mathematics, Amity University Haryana \\ Gurugram, India \\ khodiya481995@gmail.com,dba.amitsharma@gmail.com
}

Received: 9 May 2020; Accepted: 14 December 2020

\begin{abstract}
The two dimensional temperature effect on the vibration is computed for the first time for a clamped triangular plate with two dimensional thickness. In the study we focused on isosceles, right-angled and scalene triangles only. The first three modes of vibration are computed on different variations of plate parameters using the Rayleigh-Ritz method. The objective of the study is to reduce the frequency of the plates. A comparative study of the frequencies with other available results well presents the objective of the study.
\end{abstract}

MSC 2010: $74 H 45,74 K 20,93 C 80$

Keywords: temperature effect, triangle plate, frequency, vibration

\section{Introduction}

The study of plate characteristics under vibration plays an important aspects in order to understand the behaviour of engineering structures. To make an authentic model, we must know the vibration characteristics of plates. Higher in vibration/unwanted vibration can cause failure or less efficiency in the performance of the structure. In order to optimize vibration or to get desired frequencies, we have to be very careful in choosing the variations in plate parameters. The following literature study shows how plate parameters affect the vibration of plates.

The amplitude of the vibration of an equilateral, simply supported triangular plate [1] has been studied and presented graphically. Based on the Reissner-Mindlin plate theory, the vibration of triangular plates [2] with various boundary conditions is presented using the Rayleigh-Ritz method. Circular density and Poisson's ratio effect on the time period of a skew plate [3] with one dimensional thickness has been analyzed. The frequency of composite plates [4] comprises the effect of various parameters have been investigated by experiments as well as using the finite element method. Vibration of a simply supported right-angled triangle plate [5] has been presented by using a simple but accurate method. Vibration of triangle plate [6] with 
elastically restrained edges has been studied by a solution method. The Rayleigh-Ritz method has been applied to analyze the vibrations of a triangular plate [7] on various edge conditions. The atural vibration of triangle plate [8] has been studied using the the Mindlin theory, and frequencies and modal shapes have been computed on various parameters. On the basis of the Mindlin theory, the vibration of isosceles triangle plates [9] with different edge conditions using the pb-2 Rayleigh-Ritz method has been investigated. The Rayleigh-Ritz method has been used to study the vibration of a composite laminated triangle plate [10] and computed the various parameter effect on natural frequencies. Experimental as well as FEM analyses have been used to examine the vibration of a triangular plate [11] with the freeclamped-free condition. The vibration of a triangle [12] plate has been examined by using the triangular differential quadrature method, and the first six frequencies have been computed. The vibration of the triangle plate [13] with elastic edges by using the Rayleigh-Ritz method has been investigated. The Rayleigh-Ritz method has been applied to investigate the vibration of equilateral triangle [14] plates and computed the physical and geometrical parameters effect on frequencies of the plate. The vibration of a rectangle plate [15] under temperature field has been investiaged by using the Rayleigh-Ritz method. One-dimensional thermal and two dimensional thickness effect on frequency of traingle plate [16] has been computed on clamped edge conditons. Vibrational freuquency of rectangle plate $[17,18]$ comprises the nonhomogeneity, and the Poisson's ratio effect has been investigated under the temperature field. The Rayleigh-Ritz technique has been employed to investigate the frequency modes of a skew plate $[19,20]$ made of nonhomogeneous material with temperature effect.

The first objective of this study is to provide a numerical data in the form of frequencies with the help of modelling, which comprises the two-dimensional temperature and thickness effect. The second objective of the study is to minimize the frequencies, which can be well presented with the help of comparative analysis of frequency modes.

\section{Analysis}

Consider a viscoelastic triangle plate having aspect ratio $\theta=b / c$ and $\mu=c / a$ and two dimensional thickness $l$ as shown in Figure 1. Now transform the given triangle into right-angled triangle using the transformation $x=a \zeta+b \psi$ and $y=c \psi$ as shown in Figure 2.

The kinetic energy and strain energy for vibration of a traingle plate are [21]

$$
T_{s}=\frac{1}{2} \rho \omega^{2} \int_{0}^{1} \int_{0}^{1-\zeta} l \Phi^{2} a c d \psi d \zeta
$$




$$
V_{s}=\frac{1}{2} \int_{0}^{1} \int_{0}^{1-\zeta} D_{1}\left[\begin{array}{l}
\frac{1}{a^{4}}\left(\frac{\partial^{2} \Phi}{\partial \zeta^{2}}\right)^{2}+\left(\frac{b^{2}}{a^{2} c^{2}} \frac{\partial^{2} \Phi}{\partial \zeta^{2}}+\frac{1}{c^{2}} \frac{\partial^{2} \Phi}{\partial \psi^{2}}-\frac{2 b}{a c^{2}} \frac{\partial^{2} \Phi}{\partial \zeta \partial}\right)^{2}\left(\frac{1}{a^{2}} \frac{\partial^{2} \Phi}{\partial \zeta^{2}}\right)\left(\frac{b^{2}}{a^{2} c^{2}} \frac{\partial^{2} \Phi}{\partial \zeta^{2}}+\frac{1}{c^{2}} \frac{\partial^{2} \Phi}{\partial \psi^{2}}-\frac{2 b}{a c^{2}} \frac{\partial^{2} \Phi}{\partial \zeta \partial \psi}\right) \\
+2(1-v)\left(-\frac{b}{a^{2} c} \frac{\partial^{2} \Phi}{\partial \zeta^{2}}+\frac{1}{a c} \frac{\partial^{2} \Phi}{\partial \zeta \partial \psi}\right)^{2}
\end{array}\right](a c) d \psi d \zeta,
$$

where $\Phi$ is the deflection function, $\omega$ is natural frequency and $D_{1}=E l^{3} / 12\left(1-v^{2}\right)$ is flexural rigidity, here $E$ and $v$ is the Young's modulus and Poisson's ratio of the plate.

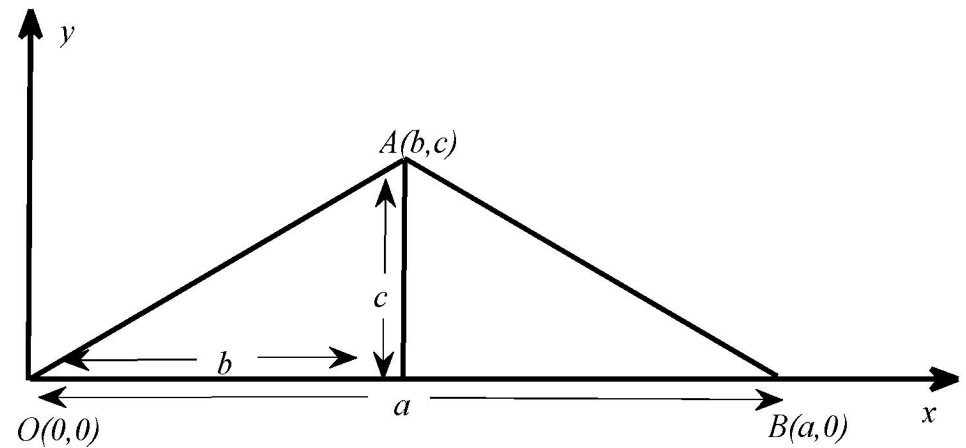

Fig. 1. Triangle plate

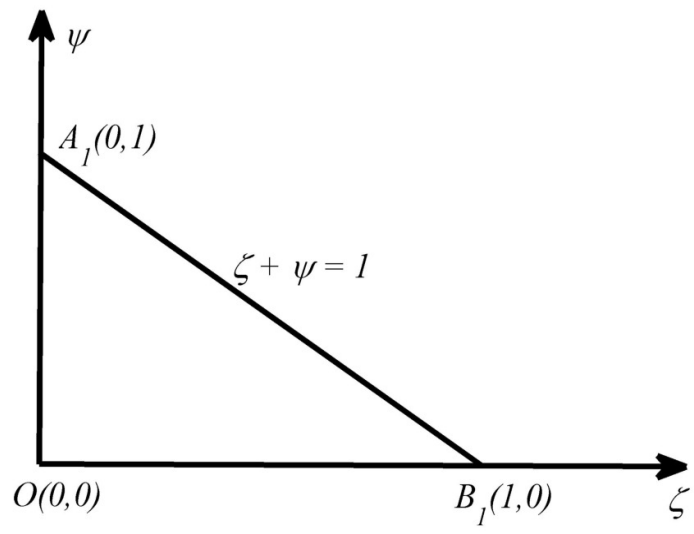

Fig. 2. Transformed triangle plate

The Rayleigh Ritz method requires

$$
L=\delta\left(V_{s}-T_{s}\right)=0 .
$$


Using Eqs. (1), (2), we have

$$
\begin{gathered}
L=\frac{1}{2} \int_{0}^{1} \int_{0}^{1-\zeta} D_{1}\left[\begin{array}{l}
\frac{1}{a^{4}}\left(\frac{\partial^{2} \Phi}{\partial \zeta^{2}}\right)^{2}+\left(\frac{b^{2}}{a^{2} c^{2}} \frac{\partial^{2} \Phi}{\partial \zeta^{2}}+\frac{1}{c^{2}} \frac{\partial^{2} \Phi}{\partial \psi^{2}}-\frac{2 b}{a c^{2}} \frac{\partial^{2} \Phi}{\partial \zeta \partial \psi}\right)^{2} \\
+2 v\left(\frac{1}{a^{2}} \frac{\partial^{2} \Phi}{\partial \zeta^{2}}\right)\left(\frac{b^{2}}{a^{2} c^{2}} \frac{\partial^{2} \Phi}{\partial \zeta^{2}}+\frac{1}{c^{2}} \frac{\partial^{2} \Phi}{\partial \psi^{2}}-\frac{2 b}{a c^{2}} \frac{\partial^{2} \Phi}{\partial \zeta \partial \psi}\right) \\
+2(1-v)\left(-\frac{b}{a^{2} c} \frac{\partial^{2} \Phi}{\partial \zeta^{2}}+\frac{1}{a c} \frac{\partial^{2} \Phi}{\partial \zeta \partial \psi}\right)^{2}
\end{array}\right](a c) d \psi d \zeta \\
-\frac{1}{2} \rho \omega^{2} \int_{0}^{1-\zeta} \int_{0}^{1-\zeta} l \Phi^{2} a c d \psi d \zeta
\end{gathered}
$$

Introducing two dimensional linear thickness as

$$
l=l_{0}\left(1-\beta_{1} \zeta\right)\left(1-\beta_{2} \psi\right),
$$

where $l_{0}$ are the thickness at origin. Also $\beta_{1}, \beta_{2}$ are tapering parameters.

Two dimensional temperature on the plate is assumed to be linear as

$$
\tau=\tau_{0}(1-\zeta)(1-\psi)
$$

where $\tau$ and $\tau_{0}$ denote the temperature on and at the origin respectively. The modu-

lus of elasticity is given by

$$
E=E_{0}(1-\gamma \tau)
$$

where $E_{0}$ is the Young's modulus at $\tau=0$, and $\gamma$ is called the slope of variation. Using Eq. (6), Eq. (7) becomes

$$
E=E_{0}(1-\alpha(1-\zeta)(1-\psi))
$$

where $\alpha=\gamma \tau_{0},(0 \leq \alpha<1)$ is called thermal gradient.

Using Eqs. (5) and (8), the functional in Eq. (4) becomes 


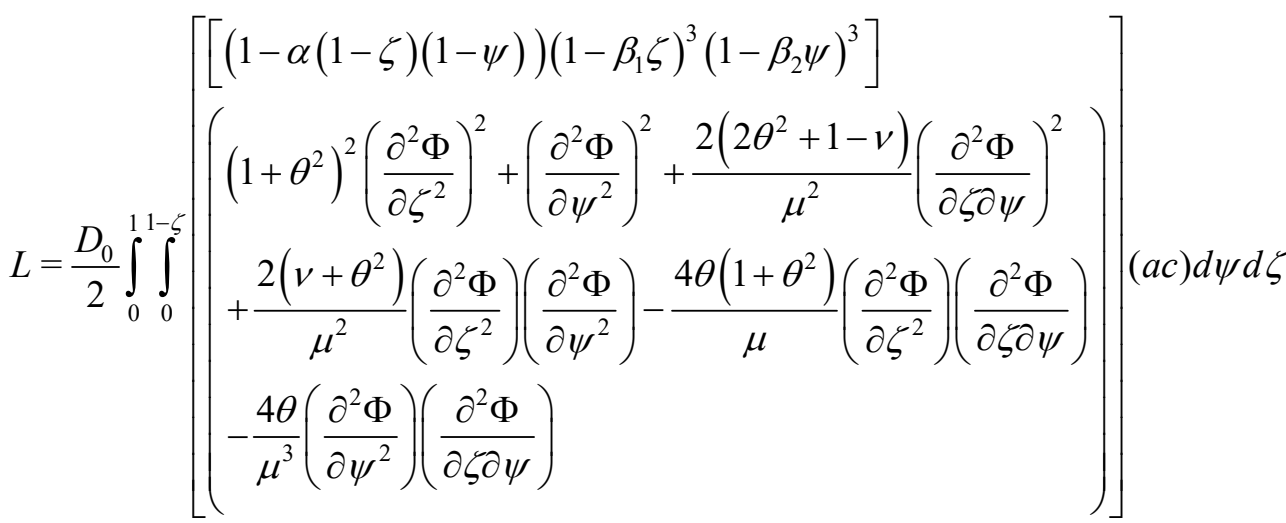

$-\frac{1}{2} \rho \omega^{2} \int_{0}^{1} \int_{0}^{1-\zeta}\left(1-\beta_{1} \zeta\right)\left(1-\beta_{2} \psi\right) \Phi^{2} a c d \psi d \zeta$

where $D_{0}=E_{0} l_{0}^{3} / 12\left(1-v^{2}\right)$ and $\lambda^{2}=\rho \omega^{2} l_{0} a^{2} / D_{0}$.

The deflection function is taken as

$$
\Phi(\zeta, \psi)=\left[(\zeta)^{e}(\psi)^{f}(1-\zeta-\psi)^{g}\right]\left[\sum_{i=0}^{n} \Psi_{i}\{(\zeta)(\psi)(1-\zeta-\psi)\}^{i}\right],
$$

where $\Psi_{i}, i=0,1,2 \ldots n$ are unknowns and the value of $e, f, g$ can be 0,1 and 2, corresponding to a given edge condition.

To minimize Eq. (10), we have

$$
\frac{\partial L}{\partial \Psi_{i}}=0, i=0,1, \ldots n
$$

Solving Eq. (11), we have frequency equation

$$
\left|P-\lambda^{2} Q\right|=0
$$

where $P=\left[p_{i j}\right]_{i, j=0,1, . . n}$ and $Q=\left[q_{i j}\right]_{i, j=0,1, . . n}$ are the square matrix of order $(n+1)$.

\section{Results and discussions}

The first three modes of vibration of isosceles, right-angled and scalene triangle plate are computed which show the effect of the thermal gradient. All the results are presented with the help of the following tables.

The first three modes of frequencies for right-angled isosceles triangle plate corresponding to a different value of taper constants with the influence of thermal 
gradient is incorporated in Table 1 . Table 1 shows that the frequency modes decrease with an increase in tapering $\beta_{1}$ and $\beta_{2}$. The temperature effect also decreases the frequency modes of the plate i.e., an increase in temperature decreases the frequencies of the plate. But the percentage rate of decrement in frequency modes increases with an increasing value of temperature on the plate.

Table 1. Frequencies of the right-angled isosceles triangle plate

\begin{tabular}{|c|c|c|c|c|c|c|c|c|c|}
\hline \multicolumn{10}{|c|}{$\theta=0, \mu=1$} \\
\hline \multicolumn{10}{|c|}{$\alpha=0.2$} \\
\hline \multirow{2}{*}{$\beta_{1}$} & \multicolumn{3}{|c|}{$\beta_{2}=0.0$} & \multicolumn{3}{|c|}{$\beta_{2}=0.4$} & \multicolumn{3}{|c|}{$\beta_{2}=0.8$} \\
\hline & $\lambda_{1}$ & $\lambda_{2}$ & $\lambda_{3}$ & $\lambda_{1}$ & $\lambda_{2}$ & $\lambda_{3}$ & $\lambda_{1}$ & $\lambda_{2}$ & $\lambda_{3}$ \\
\hline 0.0 & 102.91 & 376.80 & 854.57 & 87.37 & 320.91 & 725.60 & 74.34 & 271.81 & 613.62 \\
\hline 0.2 & 94.90 & 348.23 & 788.45 & 80.11 & 295.60 & 666.70 & 67.71 & 249.49 & 562.31 \\
\hline 0.4 & 87.37 & 320.91 & 725.54 & 73.27 & 271.43 & 612.22 & 61.43 & 228.12 & 513.97 \\
\hline 0.6 & 80.47 & 295.29 & 666.74 & 66.99 & 248.79 & 559.90 & 55.64 & 208.04 & 468.07 \\
\hline 0.8 & 74.34 & 271.80 & 613.68 & 61.43 & 228.12 & 513.18 & 50.49 & 189.70 & 427.95 \\
\hline 1.0 & 69.14 & 250.90 & 567.18 & 56.76 & 209.85 & 473.65 & 46.16 & 173.61 & 391.64 \\
\hline \multicolumn{10}{|c|}{$\alpha=0.4$} \\
\hline 0.0 & 98.60 & 360.40 & 818.49 & 83.54 & 306.36 & 693.09 & 70.87 & 258.83 & 584.44 \\
\hline 0.2 & 90.84 & 332.75 & 754.16 & 76.50 & 281.94 & 636.05 & 64.45 & 237.30 & 534.93 \\
\hline 0.4 & 83.54 & 306.36 & 693.16 & 69.87 & 258.58 & 583.38 & 58.37 & 216.65 & 488.19 \\
\hline 0.6 & 76.83 & 281.58 & 636.06 & 63.77 & 236.68 & 532.73 & 52.74 & 197.24 & 443.66 \\
\hline 0.8 & 70.87 & 258.83 & 584.49 & 58.37 & 216.66 & 487.38 & 47.73 & 179.49 & 404.69 \\
\hline 1.0 & 65.80 & 238.56 & 539.24 & 53.81 & 198.98 & 448.18 & 43.51 & 163.92 & 369.71 \\
\hline \multicolumn{10}{|c|}{$\alpha=0.6$} \\
\hline 0.0 & 94.08 & 343.12 & 780.35 & 79.51 & 291.06 & 659.18 & 67.22 & 245.16 & 553.77 \\
\hline 0.2 & 86.58 & 316.51 & 718.07 & 72.27 & 267.56 & 604.04 & 61.02 & 224.45 & 505.99 \\
\hline 0.4 & 79.51 & 291.06 & 659.16 & 66.30 & 245.05 & 553.06 & 55.13 & 204.56 & 460.83 \\
\hline 0.6 & 73.01 & 267.14 & 603.85 & 60.38 & 223.91 & 504.09 & 49.67 & 185.83 & 417.65 \\
\hline 0.8 & 67.22 & 245.16 & 553.77 & 55.13 & 204.57 & 460.03 & 44.80 & 168.66 & 380.08 \\
\hline 1.0 & 62.27 & 225.55 & 509.88 & 50.69 & 187.47 & 421.99 & 40.69 & 153.60 & 346.18 \\
\hline
\end{tabular}

Table 2 and 3 accommodate the first three modes of frequencies of right-angled scalene triangle plate corresponding to a different value of taper constants with the influence of the thermal gradient. Although the behaviour of frequency modes for the right-angled scalene triangle is the same as frequency modes of the right-angled isosceles triangle. However, the frequency modes reported in Table 2 and Table 3 are less in comparison to the frequency modes reported in Table 1 in all contexts. 
An increase in aspect ratio $\mu$ causes a decrease in the frequency modes i.e., frequency mode of right-angled scalene triangle (Tables 2 and 3) is less in comparison to frequency modes of the right-angled isosceles triangle (Table 1).

Table 2. Frequencies of the right-angled scalene triangle plate

\begin{tabular}{|c|c|c|c|c|c|c|c|c|c|}
\hline \multicolumn{10}{|c|}{$\theta=0, \mu=1.5$} \\
\hline \multicolumn{10}{|c|}{$\alpha=0.2$} \\
\hline \multirow{2}{*}{$\beta_{1}$} & \multicolumn{3}{|c|}{$\beta_{2}=0.0$} & \multicolumn{3}{|c|}{$\beta_{2}=0.4$} & \multicolumn{3}{|c|}{$\beta_{2}=0.8$} \\
\hline & $\lambda_{1}$ & $\lambda_{2}$ & $\lambda_{3}$ & $\lambda_{1}$ & $\lambda_{2}$ & $\lambda_{3}$ & $\lambda_{1}$ & $\lambda_{2}$ & $\lambda_{3}$ \\
\hline 0.0 & 92.82 & 339.88 & 770.71 & 79.23 & 291.05 & 658.81 & 67.97 & 248.61 & 563.28 \\
\hline 0.2 & 85.79 & 314.83 & 713.13 & 72.78 & 268.68 & 606.93 & 62.00 & 228.60 & 517.32 \\
\hline 0.4 & 79.23 & 291.04 & 658.93 & 66.74 & 247.38 & 559.43 & 56.36 & 209.48 & 474.16 \\
\hline 0.6 & 73.24 & 268.84 & 608.60 & 61.22 & 227.53 & 513.96 & 51.16 & 191.58 & 433.14 \\
\hline 0.8 & 67.97 & 248.60 & 563.33 & 56.36 & 209.50 & 473.25 & 46.56 & 175.26 & 397.61 \\
\hline 1.0 & 63.52 & 230.66 & 524.37 & 52.29 & 193.61 & 439.50 & 42.69 & 160.96 & 365.53 \\
\hline \multicolumn{10}{|c|}{$\alpha=0.4$} \\
\hline 0.0 & 88.85 & 324.67 & 736.94 & 75.67 & 277.52 & 628.49 & 64.73 & 236.47 & 535.79 \\
\hline 0.2 & 82.04 & 300.51 & 681.04 & 69.43 & 255.95 & 578.38 & 58.95 & 217.19 & 491.40 \\
\hline 0.4 & 75.67 & 277.52 & 628.53 & 63.57 & 235.37 & 532.43 & 53.49 & 198.76 & 449.47 \\
\hline 0.6 & 69.86 & 256.05 & 579.66 & 58.22 & 216.21 & 488.10 & 48.45 & 181.45 & 410.05 \\
\hline 0.8 & 64.73 & 236.47 & 535.73 & 53.49 & 198.74 & 449.01 & 43.98 & 165.68 & 375.57 \\
\hline 1.0 & 60.39 & 219.09 & 497.84 & 49.53 & 183.39 & 415.91 & 40.23 & 151.86 & 344.54 \\
\hline \multicolumn{10}{|c|}{$\alpha=0.6$} \\
\hline 0.0 & 84.69 & 308.71 & 701.52 & 71.94 & 263.30 & 596.67 & 61.32 & 223.67 & 506.73 \\
\hline 0.2 & 78.11 & 285.46 & 647.40 & 65.91 & 242.54 & 548.31 & 55.74 & 205.14 & 463.94 \\
\hline 0.4 & 71.94 & 263.30 & 596.64 & 60.25 & 222.75 & 503.64 & 50.46 & 187.39 & 423.59 \\
\hline 0.6 & 66.30 & 242.60 & 549.14 & 55.05 & 204.25 & 460.93 & 45.58 & 170.73 & 385.39 \\
\hline 0.8 & 61.32 & 223.67 & 506.73 & 50.46 & 187.40 & 422.99 & 41.24 & 155.50 & 352.09 \\
\hline 1.0 & 57.10 & 206.87 & 469.92 & 46.61 & 172.56 & 391.07 & 37.59 & 142.18 & 322.02 \\
\hline
\end{tabular}

The temperature effect along with tapering on the first three frequency of the scalene triangle plate is presented in Table 4. Frequency modes decrease with the increasing value of temperature on the plate. Also, an increase in both the tapering of the plate causes a decrease in the frequency modes. However, the temperature influence on the rate of decrement is noticed less in comparison to the influence of tapering parameters i.e., the rate of decrement is higher corresponding to tapering parameters in comparison to temperature. 
Table 3. Frequencies of the right-angled scalene triangle plate

\begin{tabular}{|c|c|c|c|c|c|c|c|c|c|}
\hline \multicolumn{10}{|c|}{$\theta=0, \mu=2$} \\
\hline \multicolumn{10}{|c|}{$\alpha=0.2$} \\
\hline \multirow{2}{*}{$\beta_{1}$} & \multicolumn{3}{|c|}{$\beta_{2}=0.0$} & \multicolumn{3}{|c|}{$\beta_{2}=0.4$} & \multicolumn{3}{|c|}{$\beta_{2}=0.8$} \\
\hline & $\lambda_{1}$ & $\lambda_{2}$ & $\lambda_{3}$ & $\lambda_{1}$ & $\lambda_{2}$ & $\lambda_{3}$ & $\lambda_{1}$ & $\lambda_{2}$ & $\lambda_{3}$ \\
\hline 0.0 & 89.02 & 325.93 & 739.09 & 76.17 & 279.83 & 633.96 & 65.58 & 239.94 & 544.45 \\
\hline 0.2 & 82.37 & 302.27 & 684.76 & 70.03 & 258.58 & 584.75 & 59.86 & 220.80 & 500.57 \\
\hline 0.4 & 76.17 & 279.82 & 633.98 & 64.29 & 238.38 & 539.64 & 54.46 & 202.54 & 459.21 \\
\hline 0.6 & 70.53 & 258.94 & 586.63 & 59.05 & 219.59 & 496.62 & 49.49 & 185.43 & 420.20 \\
\hline 0.8 & 65.58 & 239.95 & 544.46 & 54.46 & 202.53 & 458.68 & 45.08 & 169.87 & 386.33 \\
\hline 1.0 & 61.42 & 223.11 & 508.51 & 55.87 & 204.95 & 466.09 & 50.61 & 187.54 & 426.85 \\
\hline \multicolumn{10}{|c|}{$\alpha=0.4$} \\
\hline 0.0 & 85.17 & 311.21 & 706.07 & 72.72 & 266.69 & 604.36 & 62.43 & 228.11 & 517.64 \\
\hline 0.2 & 78.73 & 288.37 & 653.58 & 66.78 & 246.19 & 556.81 & 56.90 & 209.67 & 475.20 \\
\hline 0.4 & 72.72 & 266.69 & 604.34 & 61.21 & 226.69 & 513.25 & 51.67 & 192.04 & 435.42 \\
\hline 0.6 & 67.25 & 246.49 & 558.60 & 56.14 & 208.55 & 471.47 & 46.85 & 175.55 & 397.56 \\
\hline 0.8 & 62.43 & 228.11 & 517.61 & 51.67 & 192.04 & 434.81 & 42.58 & 160.52 & 364.70 \\
\hline 1.0 & 58.37 & 211.83 & 482.45 & 53.02 & 194.33 & 441.86 & 47.93 & 177.57 & 403.75 \\
\hline \multicolumn{10}{|c|}{$\alpha=0.6$} \\
\hline 0.0 & 81.14 & 295.74 & 671.49 & 69.10 & 252.87 & 573.08 & 59.11 & 215.63 & 489.18 \\
\hline 0.2 & 74.92 & 273.75 & 620.82 & 63.36 & 233.15 & 527.34 & 53.77 & 197.92 & 448.34 \\
\hline 0.4 & 69.10 & 252.87 & 573.06 & 57.98 & 214.39 & 485.13 & 48.72 & 180.98 & 409.71 \\
\hline 0.6 & 63.79 & 233.38 & 528.83 & 53.06 & 196.89 & 444.90 & 44.06 & 165.08 & 373.40 \\
\hline 0.8 & 59.11 & 215.64 & 489.07 & 48.72 & 180.97 & 409.26 & 39.91 & 150.58 & 341.76 \\
\hline 1.0 & 55.16 & 199.90 & 455.08 & 50.00 & 183.12 & 415.87 & 45.09 & 167.00 & 379.31 \\
\hline
\end{tabular}

Table 4. Frequencies of the scalene triangle plate

\begin{tabular}{|c|c|c|c|c|c|c|c|c|c|}
\hline \multicolumn{1}{|c|}{$\theta=1 / \sqrt{3}, \mu=\sqrt{3} / 2$} \\
\hline$\alpha$ & \multicolumn{3}{|c|}{$\beta_{1}=\beta_{2}=0.0$} & \multicolumn{3}{c|}{$\beta_{1}=\beta_{2}=0.4$} & \multicolumn{3}{c|}{$\beta_{1}=\beta_{2}=0.8$} \\
\cline { 2 - 11 } & $\lambda_{1}$ & $\lambda_{2}$ & $\lambda_{3}$ & $\lambda_{1}$ & $\lambda_{2}$ & $\lambda_{3}$ & $\lambda_{1}$ & $\lambda_{2}$ & $\lambda_{3}$ \\
\hline 0.0 & 84.94 & 311.56 & 705.52 & 65.11 & 237.38 & 539.24 & 48.82 & 175.90 & 401.75 \\
\hline 0.2 & 81.12 & 297.58 & 674.67 & 61.95 & 226.13 & 513.55 & 46.22 & 166.94 & 381.11 \\
\hline 0.4 & 77.13 & 282.96 & 641.62 & 58.63 & 214.30 & 486.49 & 43.47 & 157.47 & 359.13 \\
\hline 0.6 & 72.91 & 267.53 & 606.85 & 55.11 & 201.75 & 457.96 & 40.53 & 147.40 & 335.53 \\
\hline 0.8 & 68.43 & 251.15 & 569.99 & 51.34 & 188.37 & 427.55 & 37.35 & 136.53 & 310.41 \\
\hline
\end{tabular}


In order to understand the result discussion in a more interactive way, a graphical representation of Table 1 and Table 4 is given in Figures 3 and 4.

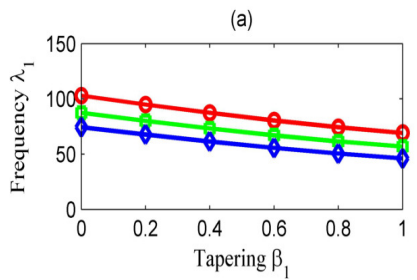

(b)
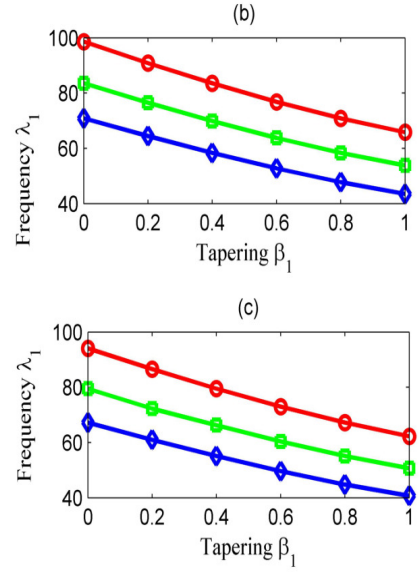

(a)

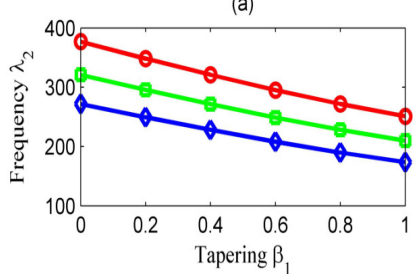

(b)

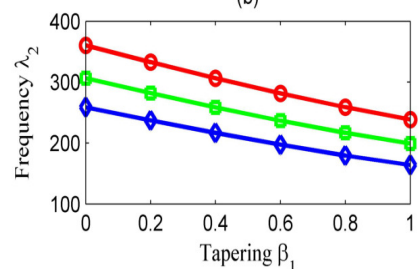

(c)

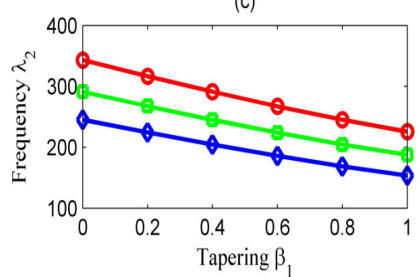

(a)

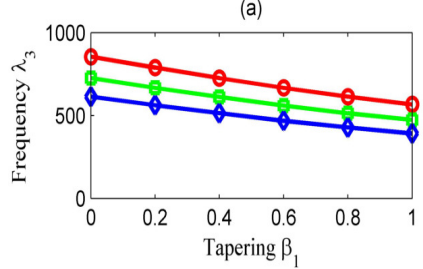

(b)

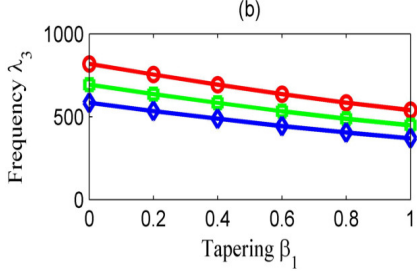

(c)

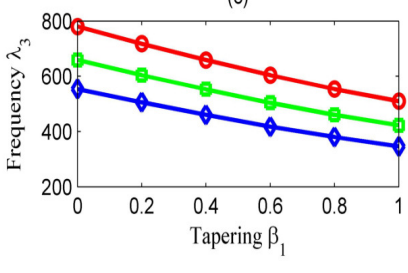

Fig. 3. Frequency $\lambda$, (a) $\alpha=0.2$; (b) $\alpha=0.4$; (c) $\alpha=0.6$ with respect to tapering $\beta_{1}$,

$-\beta_{2}=0.0,-\beta_{2}=0.4,-\beta_{2}=0.8$ for fixed value of $\theta=0, \mu=1.5$
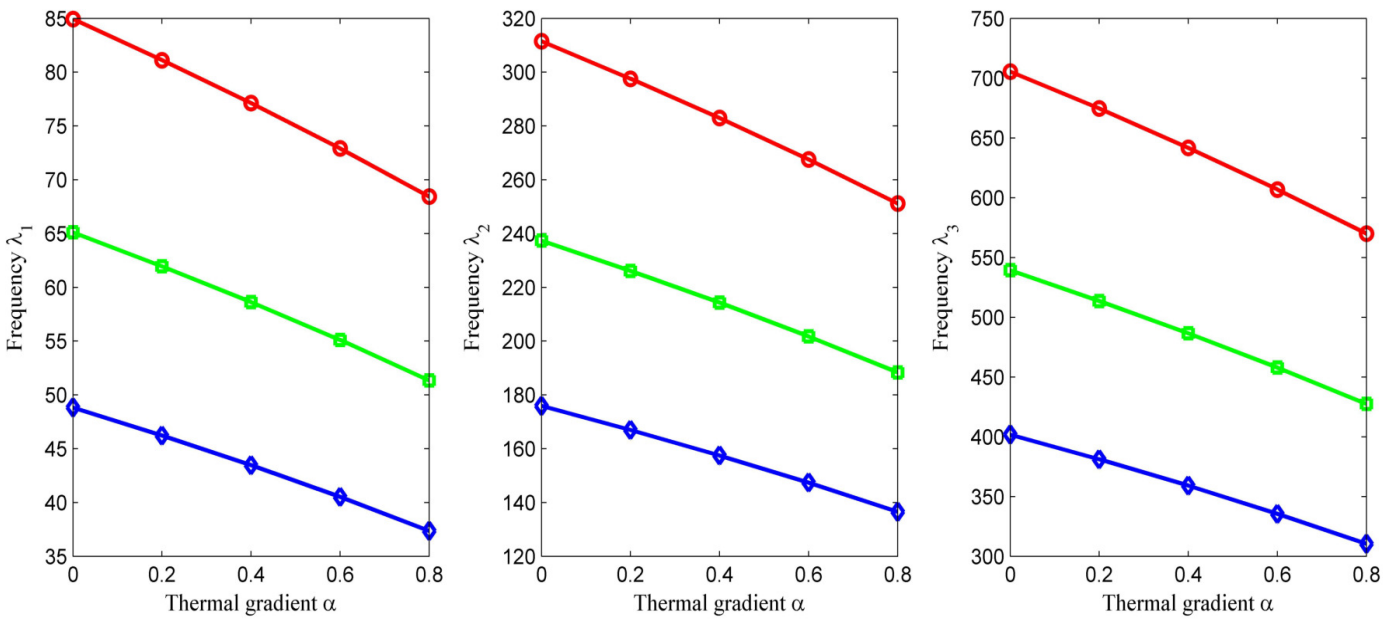

Fig. 4. Frequency $\lambda$ with respect to thermal gradient $\alpha$,

$\beta_{1}=\beta_{2}=0.0,-$

$\beta_{1}=\beta_{2}=0.4,-\beta_{1}=\beta_{2}=0.8$ for fixed value of $\theta=1 / \sqrt{3}, \mu=\sqrt{3} / 2$ 


\section{Results comparisons}

To show the validation of present study, a tabular comparison of frequency modes of the present study with an available published result is given corresponding to the thermal gradient.

Table 5 shows a comparison frequency modes (the first two modes) of the present study with [16] corresponding to a thermal gradient for a scalene triangle plate for different values of both the tapering parameters. From Table 5, it can easily be seen that the frequency modes of the present study are less when compared with the frequency obtained in [16] for the increasing values of thermal gradient on different values of tapering parameters.

Table 5. Comparison of frequency modes of the scalene triangle plate of the present study with [16]

\begin{tabular}{|c|c|c|c|c|c|c|c|c|c|c|}
\hline \multicolumn{10}{|c|}{$\theta=1 / \sqrt{3}, \mu=\sqrt{3} / 2$} \\
\hline \multirow{2}{*}{$\alpha$} & \multicolumn{2}{|c|}{$\beta_{1}=\beta_{2}=0.0$} & \multicolumn{2}{|c|}{$\beta_{1}=\beta_{2}=0.2$} & \multicolumn{2}{|c|}{$\beta_{1}=\beta_{2}=0.4$} & \multicolumn{2}{|c|}{$\beta_{1}=\beta_{2}=0.6$} & \multicolumn{2}{c|}{$\beta_{1}=\beta_{2}=0.8$} \\
\cline { 2 - 12 } & $\lambda_{1}$ & $\lambda_{2}$ & $\lambda_{3}$ & $\lambda_{4}$ & $\lambda_{1}$ & $\lambda_{2}$ & $\lambda_{3}$ & $\lambda_{4}$ & $\lambda_{1}$ & $\lambda_{2}$ \\
\hline \multirow{2}{*}{0.0} & 84.94 & 311.56 & 74.59 & 272.90 & 65.11 & 237.38 & 56.50 & 205.03 & 48.82 & 175.90 \\
& $84.96^{\mathrm{a}}$ & $346.11^{\mathrm{a}}$ & $74.61^{\mathrm{a}}$ & $304.12^{\mathrm{a}}$ & $65.13^{\mathrm{a}}$ & $265.85^{\mathrm{a}}$ & $56.53^{\mathrm{a}}$ & $231.43^{\mathrm{a}}$ & $48.85^{\mathrm{a}}$ & $201.12^{\mathrm{a}}$ \\
\hline \multirow{2}{*}{0.2} & 81.12 & 297.58 & 71.12 & 260.36 & 61.95 & 226.13 & 53.64 & 194.98 & 46.22 & 166.94 \\
& $81.15^{\mathrm{a}}$ & $330.78^{\mathrm{a}}$ & $71.14^{\mathrm{a}}$ & $290.05^{\mathrm{a}}$ & $61.98^{\mathrm{a}}$ & $252.93^{\mathrm{a}}$ & $53.67^{\mathrm{a}}$ & $219.54^{\mathrm{a}}$ & $46.25^{\mathrm{a}}$ & $190.14^{\mathrm{a}}$ \\
\hline \multirow{2}{*}{0.4} & 77.13 & 282.96 & 67.47 & 247.17 & 58.63 & 214.30 & 50.62 & 184.37 & 43.47 & 157.47 \\
& $77.15^{\mathrm{a}}$ & $314.71^{\mathrm{a}}$ & $67.50^{\mathrm{a}}$ & $275.26^{\mathrm{a}}$ & $58.65^{\mathrm{a}}$ & $239.31^{\mathrm{a}}$ & $50.64^{\mathrm{a}}$ & $206.97^{\mathrm{a}}$ & $43.50^{\mathrm{a}}$ & $178.49^{\mathrm{a}}$ \\
\hline \multirow{2}{*}{0.6} & 72.91 & 267.53 & 63.62 & 233.24 & 55.11 & 201.75 & 47.40 & 173.10 & 40.53 & 147.40 \\
& $72.93^{\mathrm{a}}$ & $297.78^{\mathrm{a}}$ & $63.64^{\mathrm{a}}$ & $259.63^{\mathrm{a}}$ & $55.13^{\mathrm{a}}$ & $224.86^{\mathrm{a}}$ & $47.42^{\mathrm{a}}$ & $193.58^{\mathrm{a}}$ & $40.55^{\mathrm{a}}$ & $166.02^{\mathrm{a}}$ \\
\hline \multirow{2}{*}{0.8} & 68.43 & 251.15 & 59.51 & 218.42 & 51.34 & 188.37 & 43.94 & 161.05 & 37.35 & 136.53 \\
& $68.45^{\mathrm{a}}$ & $279.81^{\mathrm{a}}$ & $59.53^{\mathrm{a}}$ & $242.99^{\mathrm{a}}$ & $51.36^{\mathrm{a}}$ & $209.42^{\mathrm{a}}$ & $43.95^{\mathrm{a}}$ & $179.20^{\mathrm{a}}$ & $37.36^{\mathrm{a}}$ & $152.53^{\mathrm{a}}$ \\
\hline
\end{tabular}

${ }^{\text {a }}$ Value obtained from [16]

\section{Conclusions}

The first three frequency modes of the clamped triangle plate of a different type is computed which shows the effect of thermal gradient along with tapering parameters. From results discussions and comparison, the authors would like to record the following facts:

1. Frequency modes are less in case of two dimensional linear temperature variation (present study) when compared to one dimensional linear temperature variation [16] i.e., the two dimensional temperature affects the frequencies more in comparison to one dimensional temperature. This shows that by choosing an appropriate variation in the plate parameter, the frequency can be minimized. 
2. The rate of decrement in frequency modes increases with the increasing value of the thermal gradient. The rate of decrement in the case of two dimensional temperature variations is less in comparison to the rate of decrement in the case of two dimensional tapering parameters. This shows that variation on frequencies can be controlled by choosing the appropriate value/variation of plate parameters.

3. The frequencies obtained for the right-angled isosceles triangle plate is more than the frequencies obtained for the right-angled scalene triangle plate.

4. An increase in the value of an aspect ratio together with the thermal gradient decreases the frequencies of right-angled scalene triangle plate.

\section{References}

[1] Chernyshov, N.A., \& Chernyshov, A.D. (2001). Viscoelastic vibrations of a triangular plate. Journal of Applied Mechanics and Technical Physics, 42(5), 510-515.

[2] Karunasena, W., \& Kitipornchai, S. (1997). Free vibration of shear-deformable general triangular plates. Journal of Sound and Vibration, 199, 595-613.

[3] Bhardwaj, R., \& Mani, N. (2019). Modelling on vibration of skew plate with thickness and temperature variation. Vibroengineering Procedia, 22, 6-12.

[4] Venkateshapp, S.C., Kumar, P., \& Ekbote, T. (2019). Free vibration studies on plates with central cut-out. CEAS Aeronautical Journal, 10, 623-632.

[5] Saliba, H.T. (1990). Transverse free vibration of simply supported right triangular thin plates: a highly accurate simplified solution. Journal of Sound and Vibration, 139(2), 289-297.

[6] Zhang, X.F., \& Li, W.L. (2015). Vibration of arbitrarily-shaped triangular plates with elastically restrained edges. Journal of Sound and Vibration, 357, 195-206.

[7] Singh, B., \& Chakraverty, S. (1992). Transverse vibration of triangular plates using characteristic orthogonal polynomials in two variables. International Journal of Mechanical Sciences, 34(12), 947-955.

[8] Mirza, S., \& Alizadeh, Y. (1994). Free vibration of partially supported triangular plates. Computers \& Structures, 51(2), 143-150.

[9] Kitipornchai, S., Liew, K.M., Xiang, Y., \& Wang, C.M. (1993). Free vibration of isosceles triangular Mindlin plates. International Journal of Mechanical Science, 35, 89-102.

[10] Abrate, S. (1996). Vibration of point supported triangular plates. Computers \& Structures, 58, 327-336.

[11] Chaudhary, R.R., \& Falak, Y.R. (2015). Vibration analysis of laminated triangular plate by experimental and finite element analysis. International Journal of Engineering Research and General Sciences, 3(2), 786-791.

[12] Zhong, H.Z. (2000). Free vibration analysis of isosceles triangular Mindlin plates by the triangular differential quadrature method. Journal of Sound and Vibration, 237, 697-708.

[13] Nallim, L.G., Luccioni, B.M., \& Grossi, R.O. (2005). Vibration of general triangular composite plates with elastically restrained edges. Thin-Walled Structures, 43(11), 1711-1745.

[14] Pradhan, K.K., \& Chakraverty, S. (2016). Natural frequencies of equilateral triangular plates under classical edge supports. Symposium on Statistical and Computational Modelling with Applications, 30-34.

[15] Sharma, A., Raghav, A.K., Sharma, A.K., \& Kumar, V. (2016). A modelling on frequency of rectangle plate. International Journal of Control Theory of Applications, 9, 272-282.

[16] Kaur, N. (2020). Vibrational 47ehaviour of tapered triangular plate with clamped ends under thermal condition. Journal of The Institution of Engineers (India): Series C, 1-9. 
[17] Sharma, A., Mani, N., \& Bhardwaj, R. (2019). Natural vibration of tapered rectangle plate with exponential variation in non homogeneity. Journal of Vibroengineering, 21, 187-197.

[18] Sharma, A. (2019). Vibration frequency of a rectangle plate with linear variation in thickness and circular variation in Poisson's ratio. Journal of Theoretical and Applied Mechanics, 57(3), 605-615.

[19] Lather, N., \& Sharma, A. (2019). Natural vibration of skew plate on different set of boundary conditions with temperature gradient. Vibroengineering Procedia, 22, 74-80.

[20] Sharma, A. (2019). Natural vibration of parallelogram plate with circular variation in density. Acta Technica, 63(6), 763-774.

[21] Chakraverty, S. (2009). Vibration of Plates. Boca Raton, London, New York: CRC Press, Taylor and Francis Group. 